\title{
Double Primary Brain Tumors of Different Histology in the Same Patient
}

\author{
Mithilesh Kumar Pandey ${ }^{1}$ Parag Agarwal ${ }^{1}$ Debajit Ray Barman ${ }^{1}$ Kaushik Roy ${ }^{1}$ \\ 1 Department of Neurosurgery, Nil Ratan Sirkar Medical College and \\ Hospital, Kolkata, West Bengal, India \\ Indian J Neurosurg 2015;4:173-176.

\begin{abstract}
Address for correspondence Dr. Mithilesh Kumar Pandey, MBBS, MS MCh, Department of Neurosurgery, Nil Ratan Sirkar Medical College and Hospital, Kolkata, West Bengal 700014, India (e-mail: pandeymithilesh49@gmail.com).
\end{abstract}

\begin{abstract}
Keywords

- double tumors

- meningioma

- glioblastoma multiforme

- primary brain tumor

The simultaneous occurrence of primary brain tumors of different histological type in same patient is very rare. Most of these tumors arise after cranial radiotherapy or in association with familial tumor syndromes and very few simultaneously occurring cases have been reported previously. We describe an additional case with simultaneous meningioma and glioblastoma multiforme at different intracranial location, without past cranial radiotherapy or phacomatosis in a 59-year-old female patient. Clinical manifestations, characteristic imaging findings, and possible mechanisms, as well as the treatment results are discussed and compared with previously reported cases.
\end{abstract}

\section{Introduction}

An association with multiple primary intracranial tumors of different histological types not relating to radiotherapy or phacomatosis is a rare event ${ }^{1}$ and a significant number of these occur after cranial radiotherapy ${ }^{2}$ or in phacomatosis, such as von Recklinghausen neurofibromatosis. ${ }^{3}$

The incidence of primary brain tumors with different histological types is only $0.3 \%$ of all brain tumors. ${ }^{4}$ The most frequent reported combination of histologically different brain tumors is meningioma and glioma, ${ }^{5}$ followed by meningioma and neurinoma, ${ }^{5,6}$ and by meningioma and pituitary adenoma. ${ }^{6}$ The true incidence of this association may be much higher.

\section{Case History}

A 59-year-old female patient was admitted with complains of early morning headache, focal fits affecting left upper limb, and progressive weakness of left half of the body for last 2 months. She was also complained of forgetfulness, decreased perception of smell. There was no history of tuberculosis, any systemic malignancy. Patient was alert, conscious, and cooperative. She had anosmia, bilateral temporal hemianopia, upper motor neuron type seventh cranial nerve palsy, and left-sided spastic hemiparesis with planter extensor on left side. The computed tomographic (CT) scan brain both plain and contrast showed a solid cystic lesion of size $50 \mathrm{~mm} \times 46 \mathrm{~mm}$ in right frontoparietal region and perilesional edema with heterogeneous contrast enhancement and another extra-axial lesion of size $25 \mathrm{~mm} \times 20 \mathrm{~mm}$ in basifrontal region with intense

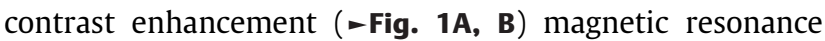
imaging (MRI) brain contrast revealed a heterogeneously enhancing mass lesion in right frontoparietal region with mass effect suggestive of high-grade glioma and another an extra-axial lesion seen in basifrontal region with pressure over both the frontal lobes suggestive of meningioma (-Fig. 2A-E) MR angiography study was normal. The right parasagittal frontoparietal craniotomy with gross total removal of basifrontal lesion with coagulation of the dural attachment (Simpson grade II), and decompression of right parietal tumor was done. Histopathologic examination of the basifrontal lesion and right parietal lesion came out to be meningothelial meningioma and glioblastoma multiforme, respectively (-Fig. 3A, B).

\section{Discussion}

The occurrence of multiple intracranial tumors of different cell types is not extremely rare but is usually observed in received

April 6, 2015

accepted

September 22, 2015

published online

December 16, 2015
DOI http://dx.doi.org/

10.1055/s-0035-1569002. ISSN 2277-954X. (c) 2015 Neurological Surgeons' Society of India
License terms

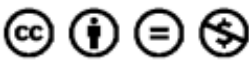



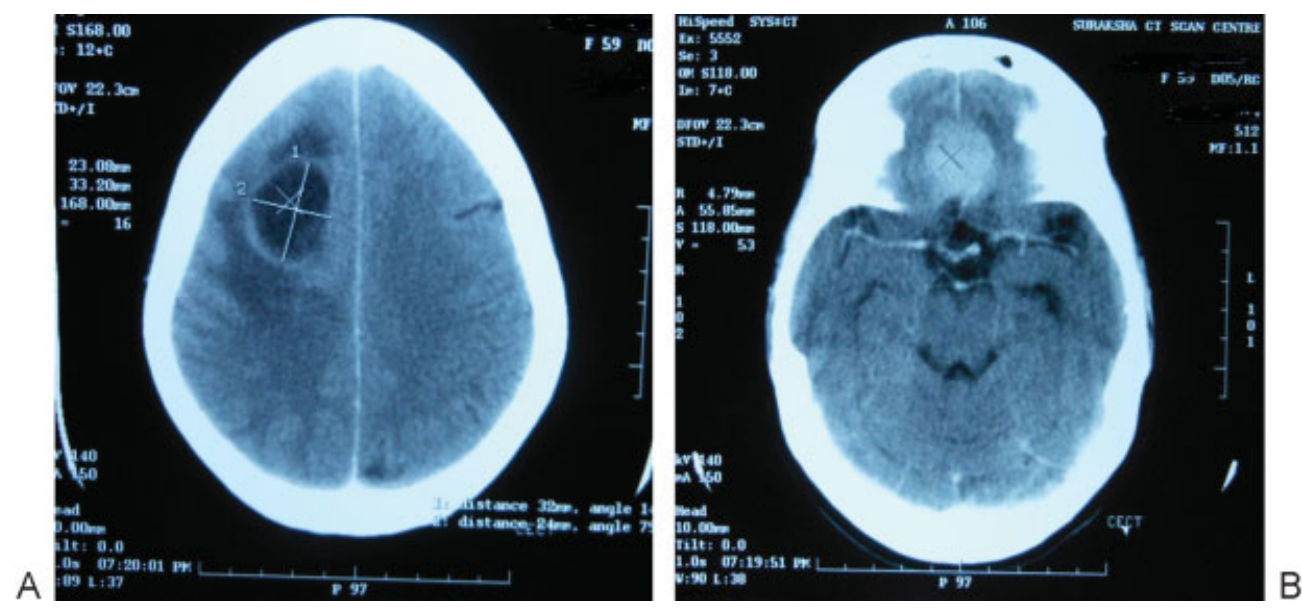

Fig. 1 (A, B) CT scan brain with contrast was showed a solid cystic lesion in right frontoparietal region and perilesional edema with heterogeneous contrast enhancement and another extra-axial lesion in basifrontal region with intense contrast enhancement. CT, computed tomography.

several genetic disorders, for example, neurofibromatosis and tuberous sclerosis, or in the case of secondary tumors formed after cranial radiotherapy. ${ }^{7}$ In fact, several reports have described coexisting meningiomas and gliomas. ${ }^{1,8,9}$

Several hypotheses have been proposed to explain the simultaneous occurrence of two (or more) primary intracranial tumors of different germinal origins in the same individual unrelated to radiotherapy or phacomatosis, but none have gained conclusive support. ${ }^{8}$ Concurrent glioma and meningioma have been reported by several authors, some of whom have concluded that this phenomenon was most likely a random statistical coincidence rather than the result of common pathway abnormalities. A nonadjacent tumoral relationship in the present case could not be explained by the notion that either of the two tumors might act as an irritating agent for the formation of the other. ${ }^{1}$
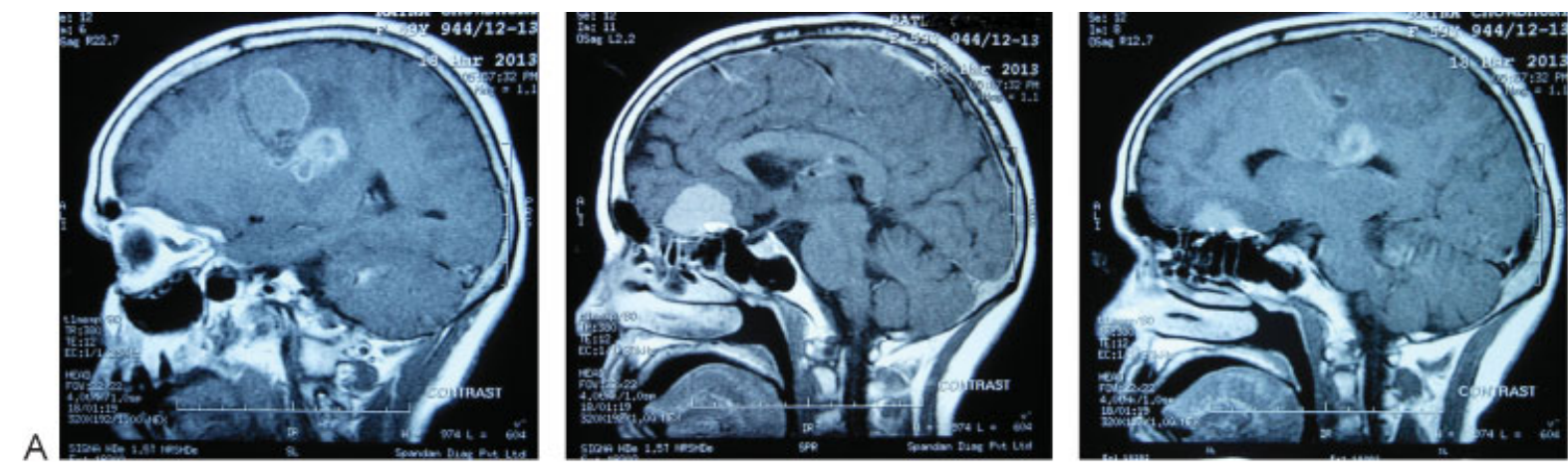

$B, C$
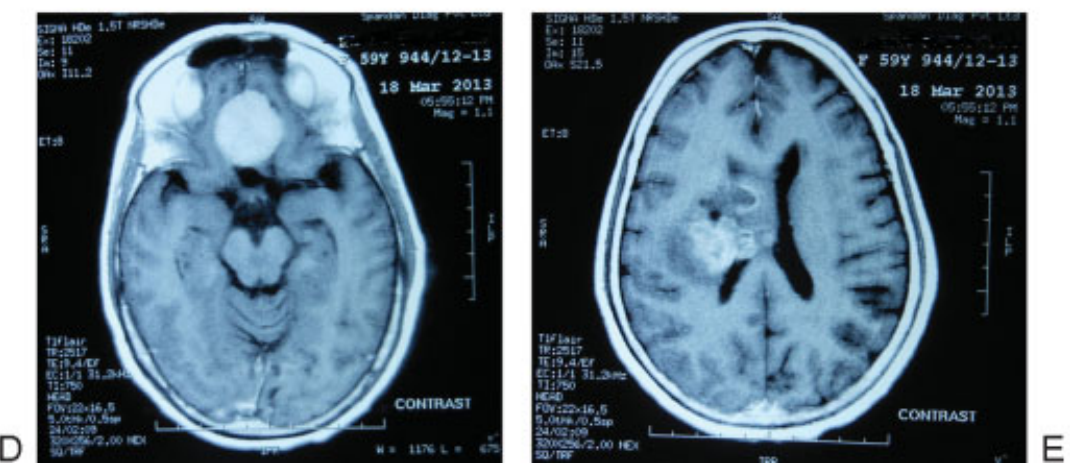

Fig. 2 (A, B) T1W with gadolinium enhanced MRI brain sagittal images was showed heterogeneously enhancing lesion with some peripheral enhancement in frontoparietal region with mass effect and another homogeneously enhancing lesion at basifrontal region with dural tail. (C) Both lesions were seen in same sagittal image. (D, E) T1FLAIR with gadolinium-enhanced MRI brain axial images was showed homogeneously enhancing lesion at basifrontal region and another enhancing lesion in right frontoparietal region with mass effect. MRI, magnetic resonance imaging; T1FLAIR; T1-weighted-fluid-attenuated inversion recovery; T1W, T1-weighted. 


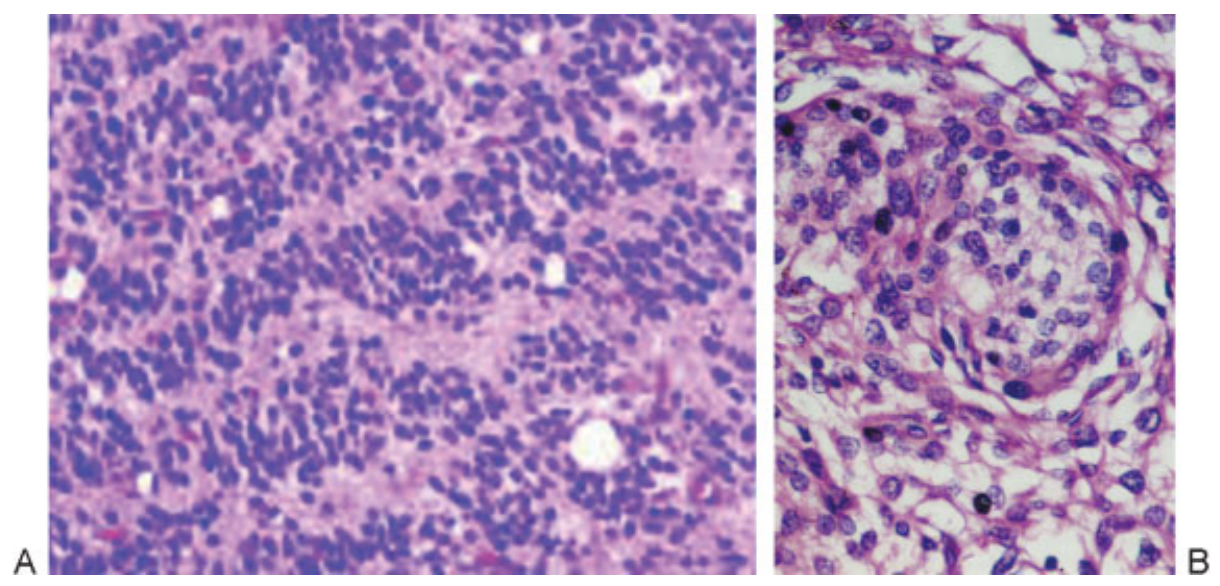

Fig. 3 (A, B) The basifrontal lesion on hematoxylin-eosin $(\times 400)$ stained was showed features of meningothelial meningioma having lobules and whorls of epithelioid cells. The right frontoparietal lesion on hematoxylin-eosin $(\times 400)$ stained showed glioblastoma multiforme having hyperchromatic nuclei and necrosis.

This pattern of tumoral association most likely represented either a purely coincidental event or some remote inciting molecular or genetic agent it is still a matter of debate. Immunohistochemical analysis revealed divergent differentiation in glioblastoma multiformes and the activation of the signaling pathways in both tumors. Dysfunction of p53 was also suggested in both tumors and this was considered to promote the progression of these tumors. $^{7}$

In our case, immunohistochemistry and molecular study was not done due to financial constraints.

Possible mechanisms for the development of multiple brain tumors with different histological types, apart from previous irradiation or phacomatosis, are mentioned below $^{10}$ :

1. Entirely coincidental development of the tumors

2. The initial tumor acts as a stimulus on the surrounding cerebral parenchyma or meningeal tissue to induce a new tumor in a different tissue

3. A carcinogenic stimulus develops tumors in different tissues simultaneously

4. A residual embryonic structure becomes the basis for subsequent multiple brain tumor development

The recent advance in neuroimaging for the diagnosis of brain tumor has resulted in more reports of multiple primary brain tumors but still it is very much difficult to see the both tumors simultaneously in a single cut section image of CT/MRI.

An important aspect in dealing with such a case is to decide which tumor should be operated first or whether both in one session. Standard protocol is that the lesion causing the main neurological symptoms should be removed first and foremost, whether it is a meningioma or an astrocytoma. $^{8}$

In contrast to this, some have suggested that one should remove the meningioma first or both the tumors in single session, provided that postoperative brain edema is avoided during the resection. ${ }^{1}$
Another controversial issue is the need of surgical resection versus conservative management to treat benign lesions in patients who have a coexistent malignant lesion. It should be emphasized that a simultaneous asymptomatic lesion, or a deep-seated and/or complex benign brain lesion which may require a more radical procedure for resection and place vital structures at risk during treatment, is not a worthwhile surgical intervention. ${ }^{1}$

One may leave the meningioma or other benign lesion in situ, thus decreasing potential morbidity caused by surgical treatment. The short expected life span of these patients may also preclude the need to remove the tumor. ${ }^{1}$ However, close follow-up and staged surgery may be necessary if the tandem lesion is thought to be an astrocytic neoplasm. ${ }^{8}$

However, we opted to resect both the tumors in a single session, for the following reasons: (1) both the tumors were easily accessible by a single approach, (2) meningioma was symptomatic and large enough in size, (3) the glioblastoma multiforme was also larger in size and cause mass effect. So the management priority for two tumors need a case-bycase evaluation.

In conclusion, the possibility of double tumor must be considered in the differential diagnosis when symptoms and signs, intraoperative findings, or unexpected postoperative events do not correlate with the preoperative radiological findings. Advanced molecular genetics techniques will be required in the future with emphasis over the importance of cell biology to understand this phenomenon of coexistence we observe in the clinical scenario.

\section{References}

1 Lee EJ, Chang $\mathrm{CH}$, Wang LC, Hung YC, Chen $\mathrm{HH}$. Two primary brain tumors, meningioma and glioblastoma multiforme, in opposite hemispheres of the same patient. J Clin Neurosci 2002;9(5):589-591

2 Domenicucci M, Artico M, Nucci F, Salvati M, Ferrante L. Meningioma following high-dose radiation therapy. Case report 
and review of the literature. Clin Neurol Neurosurg 1990;92(4): 349-352

3 Schoenberg BS. Multiple primary neoplasms and the nervous system. Cancer 1977;40(4, Suppl):1961-1967

4 Nomura K. Analysis of therapeutic factors related to survival rate for malignant glioma patients-report from Brain Tumor Registry in Japan, Vol. 6, 1987 [Article in Japanese]. Gan No Rinsho 1989; 35(1):1219-1225

5 Akagi K, Nakatani J, Ushio Y, Matsuoka K. Multiple primary brain tumors: An acoustic neurinoma associated with meningioma in the lateral ventricle. No To Shinkei 1973: 25:1823-1827

6 Kitamura K, Terao H, Kamano S, et al. Primary multiple brain tumors. No To Shinkei 1965;17:109-117
7 Suzuki K, Momota H, Tonooka A, et al. Glioblastoma simultaneously present with adjacent meningioma: case report and review of the literature. J Neurooncol 2010;99(1):147-153

8 Davis GA, Fabinyi GC, Kalnins RM, Brazenor GA, Rogers MA. Concurrent adjacent meningioma and astrocytoma: a report of three cases and review of the literature. Neurosurgery 1995; 36(3):599-604, discussion 604-605

9 Goyal A, Singh AK, Sinha S, Tatke M, Singh D, Gupta V. Simultaneous occurrence of meningioma and glioma in brain: report of two cases. J Clin Neurosci 2003;10(2):252-254

10 Tokunaga T, Shigemori M, Hirohata M, Sugita Y, Miyagi J, Kuramoto S. Multiple primary brain tumors of different histological types-report of two cases. Neurol Med Chir (Tokyo) 1991;31(3):141-145 